\title{
Creation of mathematical model optimization of Freeform Double-Layer Grids for use in the software package
}

\author{
Vadim Alpatov \\ Academy of Architecture and Civil Engineering, Samara State Technical University, Samara, Russia \\ E-mail:avu75@mail.ru
}

\begin{abstract}
It is known that metal spatial coatings can be made in shape of spatial structures, folded dual-slope structures, double mesh grid shells, double-layer braced barrel vaults, double-layer braced domes, free-form spatial structures, etc. In this paper we consider constructions on a rectangular base. The shape of the structure and distribution of material inside it are defined by solving the parametric optimization task. In the task, it has been assumed that the geometric shape of the structure can take the shape of a free-form spatial structures. A mathematical model of parametric optimization task has been presented. The task may be formulated as the discrete optimization task. The quality criterion is the minimum mass of the structure. The objective function is written through the task parameters. The analysis of permissible geometric forms of structures was made. Based on this analysis, the functions of restrictions are recorded through the parameters of the task. The restrictions are compressed by including them into objective function. As a result, a mathematical description of the objective function for the task of parametric optimization in an unconditional formulation was proposed.
\end{abstract}

Keywords: Freeform Double-Layer Grids, mathematical model, parametric optimization task.

\section{Introduction}

\section{Terms and definitions}

Structure topology - the scheme of the relative position of the rods and structure nodes.

Geometric shape - the geometric location of the nodes of the structure.

Double-layer grid spatial lattice design - spatial core design with nodes that form two mesh surfaces.

The layer grid is the surface, formed by the nodes of one of the grids of a spatial lattice structure.

The value of lifting the layer is the deviation of the center point of the belt grid from the plane formed by its corner nodes.

The value of lifting the generator of the belt grid is the deviation of the midpoint of one of the sides of the belt grid from the linear segment passing through the corner nodes.

The type of section of the structural element - the geometric shape of the cross section of the core of the structure (for example, angle, tavr, pipe, etc.).

\section{Description of counting model}

As a computational model for metal structural constructions, the hinge-rod structure is traditionally assumed to be a load applied to its nodes. The validity of this choice is confirmed by numerous experimental studies (Alpatov, 2018a, 2018b; Alpatov \& Balzannikov, 2018). However, the variety of types of nodal joints of structural structures determines the different rigidity characteristics of the nodal connectors. Accounting of rigidity of the nodal joints of structural structures can lead to material savings due to changes in the distribution of internal forces in the structure. Therefore, it is advisable in the general formulation of the problem not to narrow it within the framework of the hingerod model only, to allow the user to specify the type of node rigidity (hinged, absolutely rigid or elastic with finite rigidity).

Having two belt grids and a large number of nodes, structural structures allow numerous options for support. The bearing of structures can be performed in the corner nodes of either of the two belt grids or at all nodes of any two sides of the structure, or at the nodes of all four sides of the structure, or in some other way. This variety of support options provides freedom for researchers to determine the most advantageous support option (Chudley \& Greeno, 2007; Xu \& Wu, 2012). 
Structural structures have a high degree of static indeterminacy. The determination of the stress-strain state of spatial lattice structures is conveniently carried out numerically using computational complexes for computers.

\section{Formulation of a problem}

The task may be formulated as the following discrete optimization problem.

A spatial structural structure with two belts, with a given topology and a given geometry in the plan has been considered, the material of the elements, the conditions for fixing the structure have been determined, and the Method of Support were known. In structure the number and coordinates of the nodes, the distribution of material within the structure were unknown.

It was required to determine the geometric shape of the structure, giving it the lowest mass.

Known parameters of a task:

1) topology of construction ( $t p l)$;

2) main dimensions of construction $\left(L_{x}, L_{y}\right)$;

3) materials of elements ( $R_{y}^{i}$ - counted resistance of bar of $i$ number);

4) structure loading patterns $\left(N_{\text {load }}\right)$.

Unknown parameters of a task:

1) type of section of elements ( $t i p$ );

2) areas of cross sections of elements $\left(F_{i}\right)$;

3) number of nodes of top and bottom belt grids in structure width and length direction $\left(n_{x}^{t}, n_{y}^{t}, n_{x}^{b}, n_{y}^{b}\right)$;

4) the value of lifting the generators of the belt grid top and bottom belt grids in direction of the width and length of the construction $\left(f_{x}^{t}, f_{y}^{t}, f_{x}^{b}, f_{y}^{b}\right)$;

5) height of construction on the support $(h)$;

6) scheme of fastening the structure ( $n_{\text {sup }}$ ), that is, the nodes on which the structure will be supported.

All given and unknown characteristics of a structure, describing its condition, are called design parameters. The design parameters are divided into variable and non-variable. Variable parameters change in the process of optimizing the design, not varied remain unchanged. In turn, the variable parameters are divided into state parameters and control parameters. The former are in functional dependence on the latter, while the control parameters are independent parameters.

In our task:

- unknown parameters - $t p l, L x^{t}, L y^{t}, L x^{b}, L y^{b}, R_{y}^{i}$;

- start parameters - h, $n x^{t}, n y^{t}, n x^{b}, n y^{b}, f x^{t}, f y^{t}, f x^{b}, f y^{b}$, tip ;

- parameters of condition:

1) kinematically acceptable displacements $\left(z_{j}\right)$,

where $j \in(1,2, \ldots, m), m$-unknown movement amount ;

2) internal forces in the elements $\left(N_{i}\right)$,

where $i \in(1,2, \ldots, k), k$-amount of rods in structure;

3) cross-sectional area of elements $\left(F_{i}\right)$.

For creating a mathematical model of task, it is necessary to define the function and functions of restrictions imposed on it, through the adopted parameters.

\section{The choice of quality criteria, creation of mathematical model and description of the system of constraints}

The quality criterion is proposed to take the mass of the structure. Write the mass function of the hinge-rod structure, all elements of which are made of the same material, can be in the form:

$$
M=\gamma \times \sum_{i=1}^{n} F_{i} \times l_{n}
$$

where $i=1,2, \ldots n$ - number of element; $n$ - amount of rods in construction; $\gamma$ - specific material weight; $F_{i}-$ cross sectional area of $i$-th element; $l_{i}$ - length of $i$-th element. 
Formula (1) represents the mass of the main structural elements of construction and does not take into account the consumption of material for auxiliary parts. Auxiliary details are, for example, nodal connectors. Taking into account the mass of auxiliary elements, the mass of the whole structure can be represented as a sum:

$$
M=\sum_{i=1}^{n} m_{i}+\sum_{j=1}^{k} m_{j},
$$

where $m_{i}$ - mass of $i$-th rod of construction; $m_{j}$ - mass of $j$-th node of construction; $n$ - amount of elements in construction; $k$ - amount of nodes in construction.

First part of formula (2) shows summary mass of main elements:

$$
G_{e l m}=\sum_{i=1}^{n} m_{i}=\gamma \times \sum_{i=1}^{n} F_{i} \times l_{n} .
$$

Second part of formula (3) shows summary mass of auxilary elements:

$$
G_{a u x}=\sum_{j=1}^{k} m_{j} .
$$

In work (Trofimov \& Begun, 1972) formulas for definition of building weight coefficient for 12 types of nodes. It is suggested to count the weight of structural construction by formula:

$$
M=G_{\text {elm }} \times \Psi_{\text {weit }},
$$

where $\Psi_{\text {weit }}$ - building weight coefficient of structural construction.

Taking into account formula (3) function (5) becomes:

$$
M=\left(\gamma \times \sum_{i=1}^{n} F_{i} \times l_{n}\right) \times \Psi_{\text {weit }} .
$$

Coefficient of weight can be written as:

$$
\Psi_{\text {weit }}=(1+\rho),
$$

where $\rho$ - percent of node construction weight, for example, for construction with nodes "on tub welding" (Khisamov \& Isaeva, 1979; Trofimov \& Begun, 1972) weight of node connector equals $0.2 \div 0.25 \%$ from weight of rods. Thus $\rho=0.2 \div 0.25$ and building coefficient equals $\Psi_{\text {weit }}=1.2 \div 1.25$.

It is able to count coefficients for any other type of node like that.

Parameters of the task usually can't get random values. It is known, that:

- cross-section dimensions of construction rods must be positive;

- shape and dimensions of elements cross-sections must be chosen from available offers;

- material of elements can withstand limited stresses;

- some conditions, including constructive are contained in standards and norms of design.

This and other factors create the system of restrictions for function. The general formulation of the parametric optimization problem is mathematically written in the form of (8)

$$
f(\tilde{X})=\text { opt } f(X) \text {, where } X \in D \text {. }
$$

The problem is formulated in the form of minimization of the objective function.

$X=\left\{x_{1}, x_{2}, \ldots, x_{f}\right\}-$ set of variables of rigidity

$g_{j}(X) \leq 0 \quad(j=1, \ldots, r) \quad$ - system of restrictions: conditions of strength, rigidity, stability, structural and others imposed on the designed structure;

$f(\tilde{X})$ - scalar objective function corresponding to the accepted criterion of optimality.

Array of all $X$, answer the system of restrictions we will declare $\Omega$.

$$
\Omega=\left\{X: g_{j}(X) \leq 0, \quad j=1, \ldots, r\right\} .
$$

Then the task is:

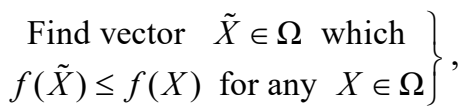

where $f$ - amount of variable parameters of construction. 
This is a task of mathematical programing about counting global extrema with restrictions.

We introduce the following restrictions: physical, geometric, structural, restrictions on changes in the geometric parameters of structures. The last group of restrictions consists of the requirements of rationality, design, architectural and aesthetic requirements. For example, the value of lifting the generator of the belt grid, must not be bigger, than length or width of the construction.

We write the restrictions through the parameters of the task.

1. Physical restrictions (strength/stability of rods):

$$
\frac{N_{i}}{F_{i}} \leq \sigma_{\alpha},
$$

where $\alpha=\operatorname{sign} \cdot R_{y i}$, that means function of design resistance of $i$-th element material, $\sigma_{+} \quad-$ for expanded elements; $\sigma_{-}-$for compressed elements.

If material of all elements is the same, then $R_{y i}=R_{y} ; \sigma_{+}=R_{y} ; \sigma_{-}=\phi \times R_{y} \quad$ - where $\phi$ is a coefficient of bending.

2. Geometrical restrictions (deformation compatibility conditions):

$$
\left.\begin{array}{l}
R_{11} \times \bar{z}_{1}+\ldots+R_{1 m} \times \bar{z}_{m}=-\bar{P}_{1 m} \\
\ldots \ldots \ldots \ldots \ldots \ldots \ldots \ldots \ldots \ldots \ldots \ldots \ldots \ldots \ldots \ldots \ldots \ldots \ldots \ldots \ldots \ldots \ldots \ldots \\
R_{n 1} \times \bar{z}_{1}+\ldots+R_{n m} \times \bar{z}_{m}=-\bar{P}_{n m}
\end{array}\right\},
$$

$R_{n m}$ - coefficients of rigidity matrix; $\bar{z}_{m}$ - single movements of node $m ; \bar{P}_{n m}$ - parts of movement method; $n-$ amount of elements; $m$ - number of unknown movements.

3. Rigidity restrictions (restriction of maximum structure main node bending):

$$
f \leq[f],
$$

where $f$ - movement of main node, $[f]$ - maximal permissible bending of structure.

4. Constructive restrictions and restrictions on geometrical parameters changes limit of structure.

a) Restriction on minimal amount of nodes in belt grids:

$$
\left.\begin{array}{ll}
n_{x}^{t} \geq\left[n_{x}^{t}\right] & n_{y}^{t} \geq\left[n_{y}^{t}\right] \\
n_{x}^{b} \geq\left[n_{x}^{b}\right] & n_{y}^{b} \geq\left[n_{y}^{b}\right]
\end{array}\right\} .
$$

These restrictions are necessary for the spatial structural structure not to degenerate into a flat structure in process of changing the parameters. Thus, the structure will stay spatial as long as the number of nodes in the belt grids of the structure in the directions of the width or length of the structure is at least two, that means: $\left[n_{x}\right]=2$ or $\left[n_{y}\right]=2$.

b) Minimal length of elements restrictions:

$$
l_{i} \geq\left[l_{\min }\right] .
$$

This restriction is necessary because the need in changing the parameters. In addition, the minimum length of the rods can be included in this restriction. For example, for nodes "on the tub welding" $\left[l_{\min }\right]$ depends on dimensions of conductor.

c) Restrictions on maximal value of lifting the generator of the belt grid:

$$
\left.\begin{array}{c}
f_{x}^{t}+f_{y}^{t} \leq L_{x} \\
f_{x}^{t}+f_{y}^{t} \leq L_{y} \\
f_{x}^{b}+f_{y}^{b} \leq L_{x} \\
f_{x}^{b}+f_{y}^{b} \leq L_{y}
\end{array}\right\},
$$


where $f_{x}^{t}$ - value of lifting the layer of top belt grid in structure width direction; $f_{y}^{t}$ - in width direction of top belt grid in structure length direction; $f_{x}^{b}-$ in width direction of bottom belt grid in structure width direction; $f_{y}^{b}-$ in width direction of bottom belt grid in structure length direction.

d) Construction height on support restrictions:

Practice of designing structural plates has shown, that optimum height values are within $\left(\frac{1}{15} \div \frac{1}{25}\right) \cdot L$ (Klyachin, 1994). It is known, that during changing height on support from $\frac{1}{24}$ to $\frac{1}{12}$ of length the weight of the structure increases.

From the experience of designing double mesh grid shells, double-layer braced barrel vaults and double-layer braced domes, it is known that their height is less than flat structures height. During the search of structural constructions optimal shape, the studied structures geometry can vary from flat structural plates to double mesh grid shells with different curvature. It is reasonable to set the maximum limit of height change on the support not more than in flat structural plates, which means $\left(\frac{1}{15}\right) \cdot L$. Minimal limit must be defined by restriction (15). Thus, this group of restrictions can be written as:

$$
\left.\begin{array}{l}
h \geq\left[l_{\min }\right] \\
h \leq\left(\frac{1}{15}\right) \cdot L
\end{array}\right\},
$$

where $L=\left\{L_{x}, L_{y}\right\}$.

e) Restrictions on unaccepting of intersection of top and bottom construction belts in length or width.

With different values of lifting the layer of top and bottom belt grids construction belt intersection is possible, which causes changes in topology of construction. (Figures 1,2). Belts intersection of structure can cause degeneration of rods (for example, diagonal members and vertical members), and they get nullable length. It is necessary to set forbiddance on changing construction topology.

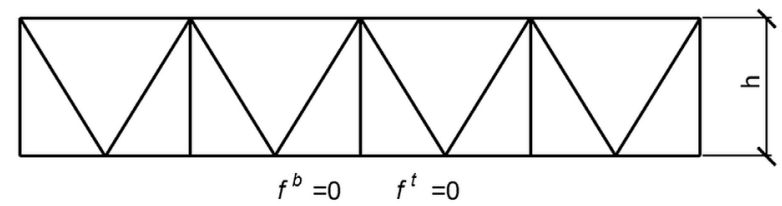

Figure 1. Flat structural plate

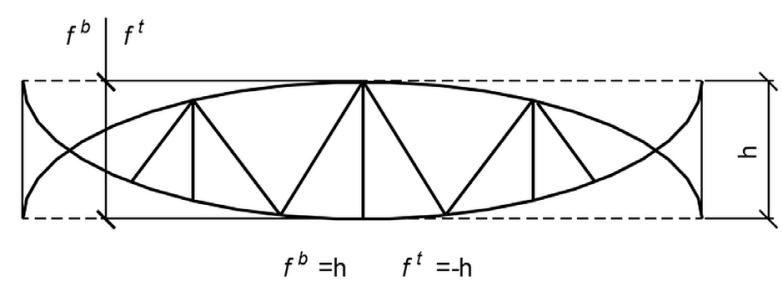

Figure 2. Degenerated structural construction with intersected belts

It is possible to prevent the intersection of the structure belts by controlling the algebraic sum of their values of lifting the layer with structure height control on the support. Consider possible cases of structural construction geometrical shape with variations of the values of lifting the layer of its top and bottom belts (Figure 3).

Value of lifting the layer is counted as sum values of lifting the generator of the belt grid in directions of width and length (18). For spatial structures values of lifting the generator of the belt grid is equal null $\left(f_{x}^{t}=0, f_{y}^{t}=0\right.$, $\left.f_{x}^{b}=0, f_{y}^{b}=0\right)$.

$$
\left.\begin{array}{l}
f_{t}=f_{x}^{t}+f_{y}^{t} \\
f_{b}=f_{x}^{b}+f_{y}^{b}
\end{array}\right\} .
$$

On Figure 3 incidents of structure degeneration (change of topology), which means events, unacceptable by task. 


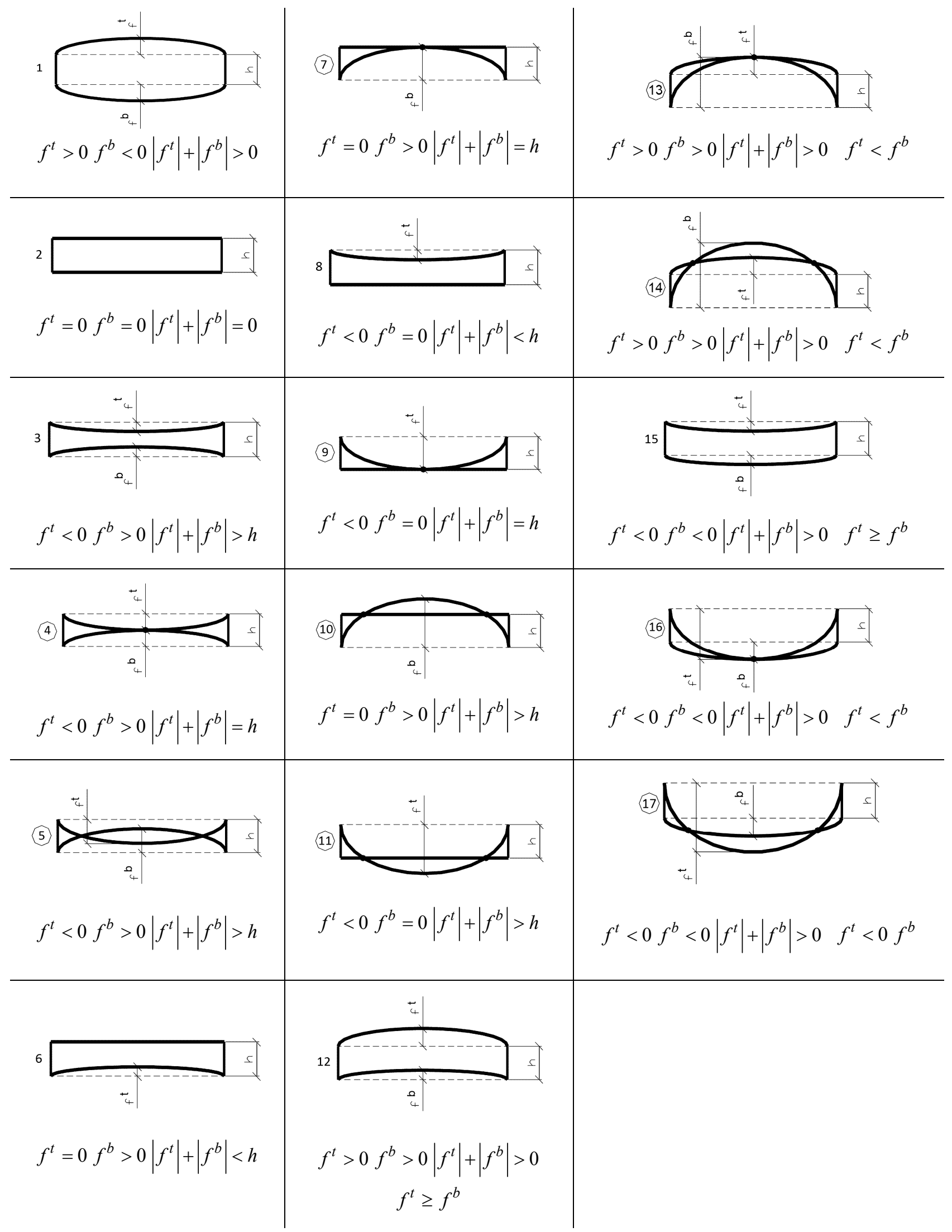

Figure 3. Possible geometrical forms of constructions with different value of lifting the layer of top and bottom belt grids 
Generalizing the possible values of lifting the layer combinations of grid belts, which were shown on images, write restrictions on avoidance of construction belts intersections as:

$$
\begin{array}{ll}
f_{t} \leq-\frac{h}{2} & f_{b} \geq \frac{h}{2} \\
f_{t}=0 & f_{b} \geq h \\
f_{t} \leq-h & f_{b}=0 \\
f_{t}>0 & f_{b}>0 \\
f_{t}<0 & f_{b}<0
\end{array}
$$

f) Restrictions on degeneration of rods (on cross-sectional area).

$$
F_{i} \leq\left[F_{\min }\right] \text {. }
$$

This group of restrictions has to save construction topology and make it geometrically unchangeable.

$\left[F_{\min }\right]$ - can be defined by requirements of maximal flexibility, minimal size profile, constructive requirements and others.

Write down target function (6) through task parameters.

Cross-sectional area is defined by its strength/stability requirements:

$$
F_{i}=\frac{\left|N_{i}\right|}{\sigma_{\alpha}} .
$$

Internal force of element can be defined using formula:

$$
N_{i}=N_{i}^{p}+\sum_{j=1}^{m} N_{i j} \times z_{j}
$$

where $j \in(1, \ldots, m) ; m$ - amount of unknown parameters; $N_{i}^{p}$ - force in $i$-th element from external load; $N_{i j}-$ force in $i$-th element from $\mathrm{j}$-th unknown parameter.

Then formula (21) becomes:

$$
F_{i}=\frac{\left|N_{i}^{p}+\sum_{j=1}^{m} N_{i j} \times z_{j}\right|}{\sigma_{\alpha}} .
$$

Length of element define through its nodes coordinates:

$$
l_{i}=\sqrt{\left(x_{i}^{b g n}-x_{i}^{e n d}\right)^{2}+\left(y_{i}^{b g n}-y_{i}^{e n d}\right)^{2}+\left(z_{i}^{b g n}-z_{i}^{\text {end }}\right)^{2}},
$$

where bgn-beginning, end - end of element.

Then formula (6) can be written as:

$$
M=\gamma \times \Psi_{\text {weit }} \times\left[\sum_{i=1}^{n}\left(\frac{\left|N_{i}^{p}+\sum_{j=1}^{m} N_{i j} \times z_{j}\right|}{\sigma^{\alpha}} \times \sqrt{\left(x_{i}^{\text {bgn }}-x_{i}^{\text {end }}\right)^{2}+\left(y_{i}^{\text {bgn }}-y_{i}^{\text {end }}\right)^{2}+\left(z_{i}^{\text {bgn }}-z_{i}^{\text {end }}\right)^{2}}\right)\right] .
$$

\section{Conclusions}

The restrictions may be compressed by including them into objective function. As a result, a mathematical description of the objective function for the task of parametric optimization in an unconditional formulation was proposed (Kholopov, Balzannikov, \& Alpatov, 2012).

Solving the problem in the described formulation allows us to obtain the geometric forms of the structures shown in Figure 4. 


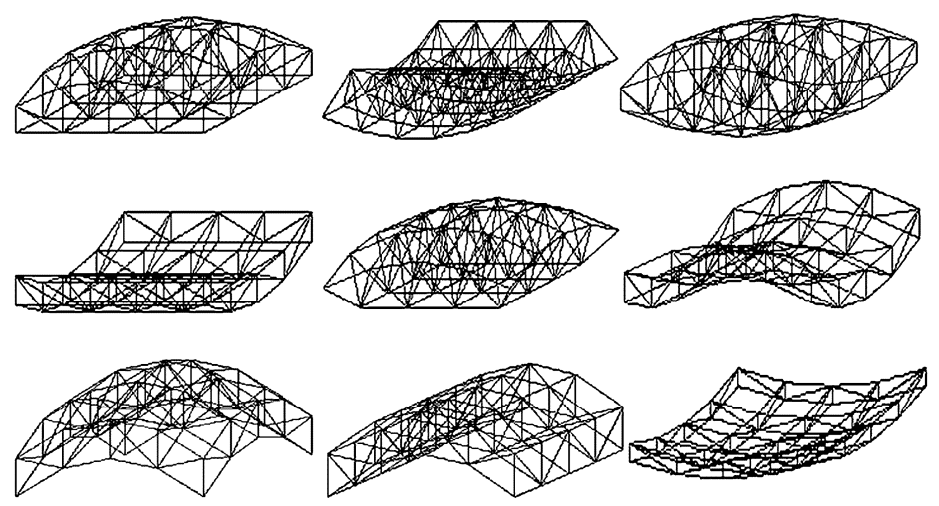

Figure 4. Possible forms of spatial constructions of structure type

\section{References}

Alpatov, V. (2018a). Numerical studies of the nodal connections of metal spatial frames. IOP Conference Series: Materials Science and Engineering, 463, 032103. https://doi.org/10.1088/1757-899X/463/3/032103

Alpatov, V. (2018b). Features of application of spatial metal farms in the production and public buildings. IOP Conference Series: Materials Science and Engineering, 463, 032102. https://doi.org/10.1088/1757-899X/463/3/032102

Alpatov, V., \& Balzannikov, M. (2018). The research of resistance of a spatial truss of a covering of the building to the progressing destruction in modern CAE-systems. 2018 IOP Conference Series: Materials Science and Engineering, 456, 012011. https://doi.org/10.1088/1757-899X/456/1/012011

Chudley, R., \& Greeno, R. (2007). Building construction handbook (6 $6^{\text {th }}$ ed.). Butterworth-Heinemann. https://www.designingbuildings.co.uk/wiki/Long_span_roof

Khisamov, R. I., \& Isaeva, L. A. (1979). Determination of technical and economic indicators of structural structures. Kazan: Kazan Institute of Chemical Technology.

Kholopov, I. S., Balzannikov, M. I., \& Alpatov, V. Yu. (2012). The use of lattice spatial metal structures in the coatings of the turbine halls. Bulletin of Volgograd State Architectural and Construction University, 28(47), 225-232.

Klyachin, A. Z. (1994). Metal lattice spatial structures of regular structure (development, research, application experience). Yekaterinburg: Diamant.

Trofimov, V. I., \& Begun, G. B. (1972). Structural structures. Moscow: Stroyizdat.

Xu, Z.-D., \& Wu, K.-Y. (2012). Damage detection for space truss structures based on strain mode under ambient excitation. Journal of Engineering Mechanics, 138(10). https://doi.org/10.1061/(ASCE)EM.1943-7889.0000426 